\title{
Value of MRI in paediatric closed head injury
}

\section{Savvas Andronikou MB BCh, FCRad (SA), FRCR (Lond)}

\section{Rudolf Boeddinghaus MB ChB}

\section{Chris J Welman MB ChB, FCRad (SA)}

Department of Paediatric Radiology Red Cross Children's Hospital Cape Town

\section{Abstract}

Children (and less commonly adults) with a low level of conciousness following acute head injury who have a normal CT scan are often assumed to have diffuse axonal injury of the brain. MRI may be appropriate in the subacute setting as it may demonstrate findings highly suggestive of such injury, as well as other findings not identified on CT. These findings may aid clinicians in determining prognosis. We demonstrate typical findings on various $\mathrm{MR}$ sequences using an index case.

A 2-year-old boy presented with a depressed level of consciousness (GCS 8/11) following a motor vehicle accident. The CT scan performed within 6 hours showed only a small subarachnoid haemorrhage, with intraventricular extension. He remained comatose, and MRI was performed after 5 days. The T2-weighted images (Figs $1 \mathrm{a}$ and $1 \mathrm{~b}$ ) showed high signal intensity in the splenium of the corpus callosum, at the insular greywhite interface on the left, in both temporo-occipital regions and in the right thalamus. It also demonstrated small bifrontal subdural collections, larger on the left. Diffusion-weighted images (Fig. 2a) demonstrated increased signal (restricted diffusion) in all of the same sites, except the right thalamus. On long-TR gradient-echo (Fig. 2b), the right thalamus showed marked hypointensity in keeping with blood breakdown products. On the basis of these findings, diffuse axonal injury, non-haemorrhagic cortical contusions, small bifrontal subdural collections and a right thalamic haemorrhage were diagnosed. None of these was evident at CT. The patient's level of consciousness improved gradually, but he had residual right hemiparesis.

Shearing injuries are common in infants and young children, because the unmyelinated brain is less rigid and the subarachnoid spaces are large. ${ }^{1}$ In the subacute phase, MR imaging is more sensitive than $\mathrm{CT}$ in detecting traumatic brain injury, such as diffuse axonal injury (DAI), nonhaemorrhagic cortical contusions, haemorrhage and surface collections. ${ }^{1.2}$ In DAI, T2-hyperintensity is found in typical locations (the corpus callosum, particularly the splenium and posterior body, the dorsolateral rostral brainstem, and hemispheric grey-white interfaces). ${ }^{3}$ Gradient-echo images are particularly helpful in demonstrating small petechial haem-
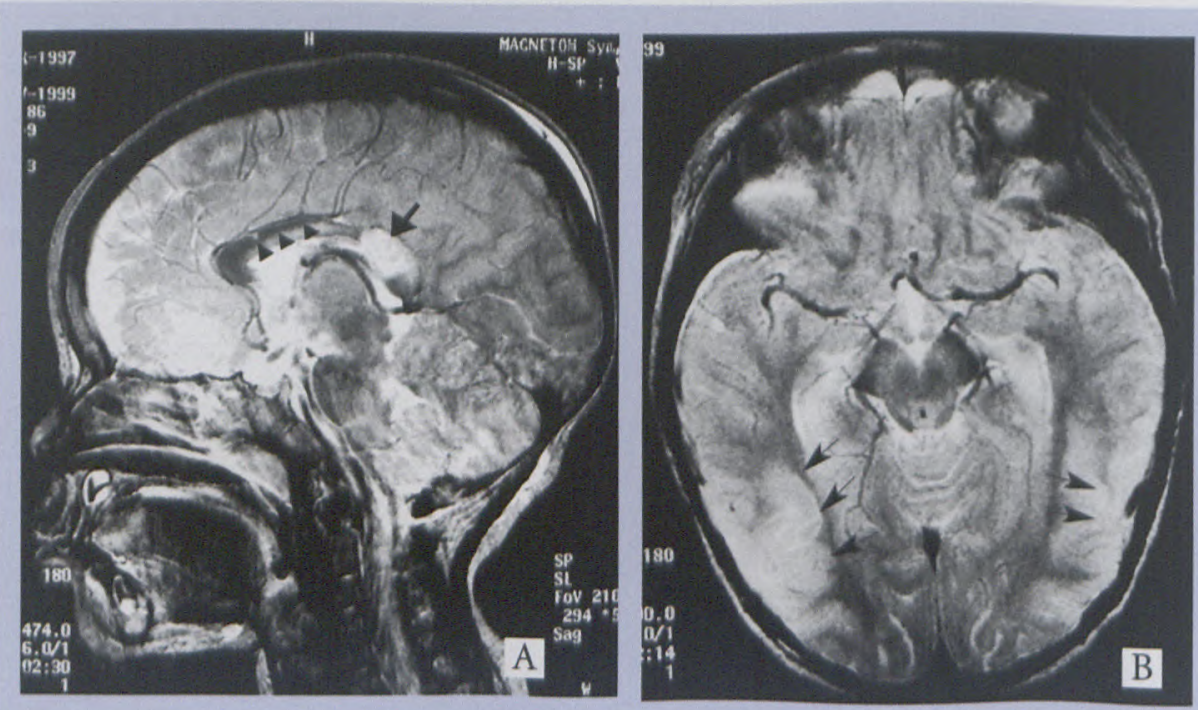

Figs ta and b. Conventional T2-weighted imaging. (a) Sagittal T2W MRI demonstrates focal high-signal lesion in the splenium of the corpus callosum (arrow) and diffuse high signal intensity throughout the body of the corpus callosum (arrowheads). The sagittal is superior to the axial plane in showing corpus callosum abnormalities. These features are characteristic of DAl. (b) Axial T2W MRI shows ill-defined bilateral occipito-temporal high-signal cortical lesions (arrows) in keeping with non-haemormagic contusions. They were not evident on CT. 


\section{CASE REPORT}
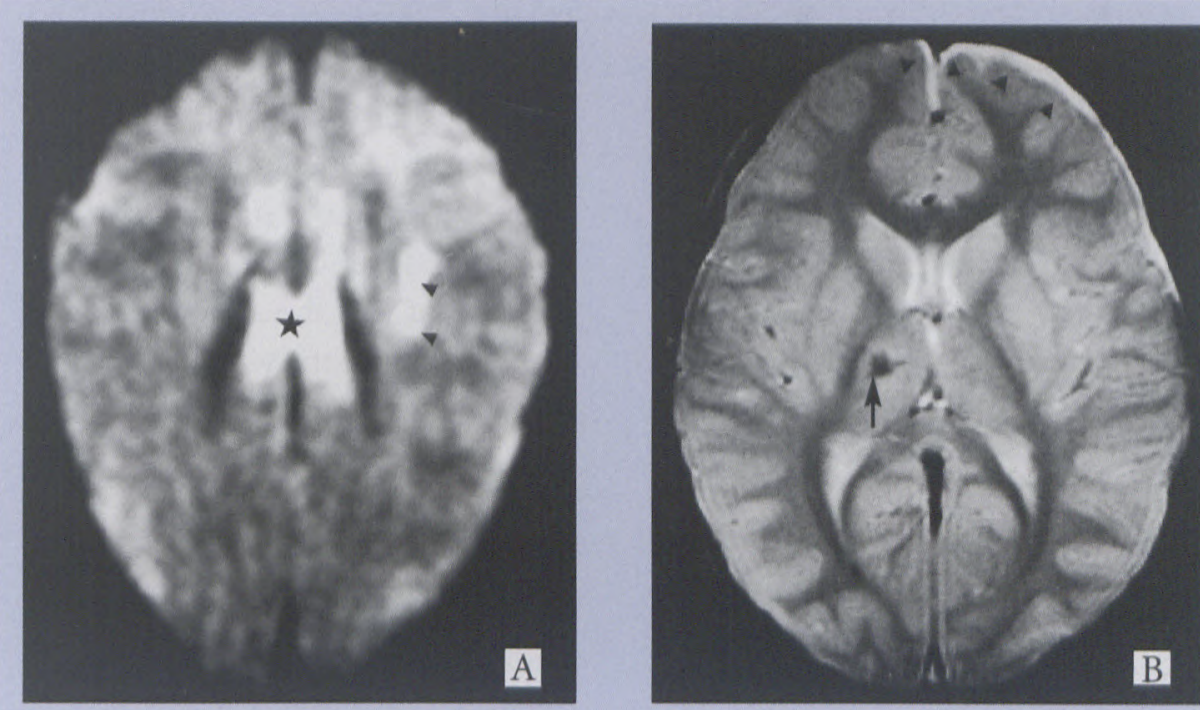

Figs 2a and b. Special MR techniques. (a) Diffusion-weighted image demonstrates high signal over the corpus callosum (star) and at the left insular grey-white interface (arrowheads) in keeping with cytotoxic oedema. These locations are characteristic of DAI. (b) Long-TR gradient echo axial image clearly demonstrates the low signal of blood breakdown products in the right thalamus (arrow). Also demonstrated are bilateral frontal subdural collections (arrowheads) that were not shown on CT. orrhages associated with DAI, with signal blooming, due to magnetic susceptibility effects. ${ }^{1,2}$ Diffusion-weighted MRI has a role to play in distinguishing cytotoxic oedema (in DAI) from vasogenic oedema (brain swelling). ${ }^{2}$ In our patient, all the above sequences showed abnormalities, which were not evident at CT. MRI is an important prognostic tool in paediatric head injury.

\section{References}

1. Barkovich AJ. Pediatric Neuroimaging. 3rd ed. Philadelphia: Lippincot Williams and Wilkins, 2000: 234-236.

2. Woodcock RJ, Davis PC, Hopkins KL. Imaging of head trauma in infancy and childhood. Semin Ultrasound CT MR 2001; 22 (2): 162182.

3. Kampfl A, Franz G, Aichner F, et al. The persistent vegetative state after closed head injury: clinical and magnetic resonance imaging findings in 42 patients. J Neurosurg 1998; 88: 809816. 